\title{
WIMAN-VALIRON THEORY IN TWO VARIABLES
}

\author{
P. C. FENTON
}

\begin{abstract}
Inequalities are obtained for the coefficients of the Taylor series of an entire function of two complex variables and used to obtain an inequality for the maximum modulus of the function in terms of the maximum term of the series.
\end{abstract}

\section{INTRODUCTION}

Some time ago W. H. J. Fuchs [3] asked whether formulations of the WimanValiron method, applicable to entire functions of a single variable, for instance, $[1,2,4,5,6,10]$, might be generalized to entire functions:

$$
f\left(z_{1}, z_{2}\right)=\sum_{n_{1}=0}^{\infty} \sum_{n_{2}=0}^{\infty} a_{n_{1}, n_{2}} z_{1}^{n_{1}} z_{2}^{n_{2}}
$$

of two variables $z_{1}$ and $z_{2}$. The essence of the Wiman-Valiron method is the analysis of the behaviour of the function by means of the maximum term:

$$
\mu\left(r_{1}, r_{2}\right)=\mu\left(r_{1}, r_{2}, f\right)=\max \left\{\left|a_{n_{1}, n_{2}}\right| r_{1}^{n_{1}} r_{2}^{n_{2}}: n_{1}, n_{2} \geq 0\right\}
$$

of its Taylor series, together with the central index: $\mathbf{N}\left(r_{1}, r_{2}\right)=\mathbf{N}\left(r_{1}, r_{2}, f\right)$, which is the pair $\left(N_{1}, N_{2}\right)$ of indices corresponding to the maximum term. ( $\mathbf{N}$ is ill-defined whenever there are several terms of maximum modulus. The central index can then be either left undefined or else fixed according to some preformulated rule, the exact nature of which is, for the most part, of no significance.) An important step is to obtain estimates for the terms of the Taylor series that adequately reflect the fact that the general term diminishes the further it is from the maximum term.

Schumitzky's thesis [8; 9], which develops Rosenbloom's probabilistic methods [7], was in part the prompt for Fuchs's question but at the same time it still constitutes, as far as I know, the most successful answer. One of the main results (in its two-variable formulation-Schumitzky deals with the general $n$-variable case) has to do with the maximum modulus of $f$ :

$$
M\left(r_{1}, r_{2}\right)=M\left(r_{1}, r_{2}, f\right)=\max _{\left|z_{1}\right| \leq r_{1},\left|z_{2}\right| \leq r_{2}}\left|f\left(z_{1}, z_{2}\right)\right| .
$$

Theorem $\mathbf{A}$ [8]. Let $f\left(z_{1}, z_{2}\right)$ be an entire function of two variables $z_{1}$ and $z_{2}$. Given $\varepsilon>0$ there is a positive constant $C$, independent of $f$, and there are

Received by the editors November 3, 1993 and, in revised form November 18, 1994.

1991 Mathematics Subject Classification. Primary 32A05. 
positive numbers $R_{1}$ and $R_{2}$, such that

$$
M\left(r_{1}, r_{2}\right) \leq C \mu\left(r_{1}, r_{2}\right)\left\{\log \mu\left(r_{1}, r_{2}\right) \log r_{1} r_{2}\right\}^{1+\varepsilon},
$$

for all $\left(r_{1}, r_{2}\right)$ outside a set $E$ such that

$$
\int_{E \cap\left[R_{1}, \infty\right) \times\left[R_{2}, \infty\right)} \frac{d r_{1} d r_{2}}{r_{1} r_{2}}<\infty .
$$

The estimate for the exceptional set here is the best one could reasonably expect, but the factor $\log r_{1} r_{2}$ on the right-hand side of (1.2) - which, Schumitzky observed, can be discarded if $f$ has nonzero lower order-is not.

The intention of this note is to give an alternative account of Wiman-Valiron theory by way of comparison series that allows, among other things, a sharpening of (1.2). The use of comparison series for functions of one variable was made systematic by Kövari [5, 6; 4], and it is an analogue of his method that forms the basis of what follows.

Exceptional sets that arise here are estimated in terms of plane logarithmic measure and upper logarithmic density, defined along the lines of their linear counterparts. Given a subset $E$ of $\mathbf{R}^{2}$ that lies in the first quadrant, its logarithmic measure is

$$
\log \operatorname{meas}(E)=\int_{E \cap(1, \infty) \times[1, \infty)} \frac{d r_{1} d r_{2}}{r_{1} r_{2}} ;
$$

and, with

$$
E_{R}=E \cap \Delta_{R}, \quad R \geq 1,
$$

where

$$
\Delta_{R}=\left\{\left(r_{1}, r_{2}\right): 1 \leq r_{1} \leq R \text { and } 1 \leq r_{2} \leq R,\right.
$$

the upper logarithmic density of $E$ is

$$
\overline{\log \operatorname{dens}}(E)=\limsup _{R \rightarrow \infty} \frac{\log \text { meas } E_{R}}{\log \text { meas } \Delta_{R}}=\limsup _{R \rightarrow \infty} \frac{\log \text { meas } E_{R}}{(\log R)^{2}} .
$$

There is also the lower logarithmic density, in which the lower limit takes the place of the upper limit in (1.5), but we have no need of it here.

In the statement of the first theorem we shall use the notation

$$
\log _{n}^{+}(t)=\log _{n}\left(\max \left\{t, e_{n}(0)\right\}\right),
$$

$\log _{n}$ being the $n$-times iterated logarithm and $e_{n}$ the $n$-times iterated exponential.

We shall prove

Theorem 1. Let $f\left(z_{1}, z_{2}\right)$ be an entire function of two variables $z_{1}$ and $z_{2}$. Given $\varepsilon>0$ and a nonnegative integer $k$, there is a constant $K=K(k, \varepsilon)$ such that

$$
\begin{aligned}
M\left(r_{1}, r_{2}\right) \leq K \mu\left(r_{1}, r_{2}\right)\left\{\left[1+N_{1} \log _{1}^{+}\right.\right. & \left.N_{1} \cdots\left(\log _{k+1}^{+} N_{1}\right)^{1+\varepsilon}\right] \\
\cdot & {\left.\left[1+N_{2} \log _{1}^{+} N_{2} \cdots\left(\log _{k+1}^{+} N_{2}\right)^{1+\varepsilon}\right]\right\}^{1 / 2}, }
\end{aligned}
$$

for all $\left(r_{1}, r_{2}\right)$ outside a set $E$ such that

$$
\int_{E_{R}} \frac{d r_{1} d r_{2}}{r_{1} r_{2}}<2(1+\varepsilon) \log R+O(1) \text { as } R \rightarrow \infty,
$$


where $\left(N_{1}, N_{2}\right)$ is the central index at $\left(r_{1}, r_{2}\right)$. Further, outside the same exceptional set,

$$
M\left(r_{1}, r_{2}\right) \leq K \mu\left(r_{1}, r_{2}\right) \log _{1}^{+} \mu\left(r_{1}, r_{2}\right)\left\{\log _{2}^{+} \mu\left(r_{1}, r_{2}\right) \cdots\left(\log _{k+2}^{+} \mu\left(r_{1}, r_{2}\right)\right)^{1+\varepsilon}\right\}^{2}
$$

As we shall see, (1.6) continues to hold if $K$ is replaced by anything greater than $2 \pi / \varepsilon$, and (1.8) if $K$ is replaced by anything greater than $2 \pi / \varepsilon^{2}$, provided that in both cases $\left(r_{1}, r_{2}\right)$ is outside $E$ and such that both $N_{1}$ and $N_{2}$ are sufficiently large. It will be shown later $(\S 6)$ that it is not possible to replace $\varepsilon$ by 0 in (1.6), but $I$ do not know if the same is true for (1.8). Certainly (1.8) does not hold in general outside a set of finite logarithmic measure, nor indeed outside a set of the kind appearing in Schumitzky's theorem. This can be seen from a function of the form $f\left(z_{1}, z_{2}\right)=F\left(z_{1}\right)$, where $F$ is an entire function of one variable for which (with $M(r, F)$ denoting the maximum modulus and $\mu(r, F)$ the maximum term)

$$
M(r, F)>\mu(r, F)\{\log \mu(r, F)\}^{2},
$$

for all $r$ in an unbounded sequence of nonempty open intervals. (Such a function can be constructed from gappy polynomial blocks of the form

$$
\frac{z^{n}}{n !}\left\{1+\frac{z}{n}+\frac{z^{2}}{n^{2}}+\cdots+\frac{z^{n^{2}}}{n^{n^{2}}}\right\} .
$$

When $z=n$ the maximum term is, if the gaps between successive blocks are large enough, i.e. if $n$ is part of a sufficiently sparse sequence, $n^{n} / n$ !, and there are $n^{2}+1$ such terms. Since $\mu(n, F)=n^{n} / n !<e^{n}$, we have $M(n, F)>n^{2} \mu(n, F)>\mu(n, F)\{\log \mu(n, F)\}^{2}$, and the same inequality continues to hold for $r$ in a small interval about $n$. Taking all blocks into account, we get $M(r, F)>\mu(r, F)\{\log \mu(r, F)\}^{2}$ for all $r$ in an unbounded sequence of nonempty open intervals.) For then $M\left(r_{1}, r_{2}\right)=M\left(r_{1}, F\right)$ and $\mu\left(r_{1}, r_{2}\right)=\mu\left(r_{1}, F\right)$ so that

$$
M\left(r_{1}, r_{2}\right)>\mu\left(r_{1}, r_{2}\right)\left\{\log \mu\left(r_{1}, r_{2}\right)\right\}^{2}
$$

for all $\left(r_{1}, r_{2}\right)$ in a set of infinite logarithmic measure, in fact on a set $E$ such that

$$
\int_{E_{R}} \frac{d r_{1} d r_{2}}{r_{1} r_{2}}>\text { (const) } \log R
$$

for all large $R$.

The inequality (1.8) can be improved if something is known about the growth of $f$. Let us say that $f$ has order $\lambda$ if

$$
\limsup _{R \rightarrow \infty} \frac{\log _{2} M(R, R)}{\log R}=\lambda \text {. }
$$

We have

Theorem 2. Suppose that $f\left(z_{1}, z_{2}\right)$ is an entire function of two variables $z_{1}$ and $z_{2}$, of finite order $\lambda$. Given $\varepsilon>0$, there is a constant $K=K(\lambda, \varepsilon)>0$ such that

$$
M\left(r_{1}, r_{2}\right) \leq 2 \pi(\lambda+\varepsilon+o(1)) \mu\left(r_{1}, r_{2}\right)\left(K+N_{1}\right)^{1 / 2}\left(K+N_{2}\right)^{1 / 2}
$$


for ail $\left(r_{1}, r_{2}\right)$ outside a set $E$ of upper logarithmic density no more than 1 $\left(\frac{\varepsilon}{\lambda+\varepsilon}\right)^{2}$, where $\left(N_{1}, N_{2}\right)$ is the central index at $\left(r_{1}, r_{2}\right)$. Further, outside the same set $E$,

$$
M\left(r_{1}, r_{2}\right) \leq\left(\pi(\lambda+\varepsilon)^{2}+o(1)\right) \mu\left(r_{1}, r_{2}\right) \log \mu\left(r_{1}, r_{2}\right) .
$$

The constant in (1.9) is, as we shall show in $\S 6$, best possible in the sense that if $\varepsilon$ is given a small negative value, then (1.9) may fail for all large $\left(r_{1}, r_{2}\right)$. For (1.10) the case is open. Nevertheless we shall show the much weaker result, that $\varepsilon$ cannot be negative if (1.10) is to hold outside a set $E$ satisfying

$$
\int_{E_{R}} \frac{d r_{1} d r_{2}}{r_{1} r_{2}}=o(\log R) \quad \text { as } R \rightarrow \infty .
$$

To the best of my knowledge Theorem 2 is the first result of its kind involving plane logarithmic densities. The methods used below are quite general. It will be evident to the reader that they can be applied to entire functions satisfying other growth restrictions, such as, for instance,

$$
\limsup _{R \rightarrow \infty} \frac{\log M(R, R)}{(\log R)^{p}}<\infty
$$

for some $p>1$.

\section{The MAIN INEQUalities}

Following Hayman $[4$, p. 321], we define the comparison series in terms of a function $\alpha(t), t \in[0, \infty)$, which is positive and increasing. The series has coefficients:

$$
\alpha_{n_{1}, n_{2}}=\alpha_{n_{1}} \alpha_{n_{2}}, \quad \text { where } \alpha_{n}=\exp \left(\int_{0}^{n}-\alpha(t) d t\right) .
$$

The only further requirement concerning $\alpha(t)$ is that it should be sufficiently large that the series

$$
F\left(z_{1}, z_{2}\right)=\sum_{n_{1}=0}^{\infty} \sum_{n_{2}=0}^{\infty} A_{n_{1}, n_{2}} z_{1}^{n_{1}} z_{2}^{n_{2}}, \quad \text { where } A_{n_{1}, n_{2}}=\frac{a_{n_{1}, n_{2}}}{\alpha_{n_{1}, n_{2}}}
$$

associated with the function (1.1) is itself an entire function. Let us suppose that this requirement has been met.

Given a pair $\left(t_{1}, t_{2}\right)$ of positive numbers, let $\left(N_{1}, N_{2}\right)$ be the central index of $F\left(t_{1}, t_{2}\right)$, so that $\left|A_{N_{1}, N_{2}}\right| t_{1}^{N_{1}} t_{2}^{N_{2}}$ is its maximum term; should there be ambiguity let us decide to choose the term for which $N_{1}+N_{2}$ is largest and, if there is more than one such term, choose the one for which $N_{1}$, say, is largest. Thus from (2.2)

$$
\frac{\left|a_{n_{1}, n_{2}}\right|}{\alpha_{n_{1}, n_{2}}} t_{1}^{n_{1}} t_{2}^{n_{2}} \leq \frac{\left|a_{N_{1}, N_{2}}\right|}{\alpha_{N_{1}, N_{2}}} t_{1}^{N_{1}} t_{2}^{N_{2}}, \quad n_{1} \geq 0, n_{2} \geq 0 .
$$

We multiply both sides by $\rho_{N_{1}}^{n_{1}-N_{1}} \rho_{N_{2}}^{n_{2}-N_{2}}$, where

$$
\rho_{n}=\exp (\alpha(n)), \quad n \geq 0,
$$

and obtain, after rearrangement,

$$
\frac{\left|a_{n_{1}, n_{2}}\right|}{\left|a_{N_{1}, N_{2}}\right|} r_{1}^{n_{1}-N_{1}} r_{2}^{n_{2}-N_{2}} \leq \frac{\alpha_{n_{1}, n_{2}}}{\alpha_{N_{1}, N_{2}}} \rho_{N_{1}}^{n_{1}-N_{1}} \rho_{N_{2}}^{n_{2}-N_{2}}, \quad n_{1} \geq 0, n_{2} \geq 0,
$$


where $r_{1}=t_{1} \rho_{N_{1}}$ and $r_{2}=t_{2} \rho_{N_{2}}$. The right-hand side of (2.4) is, taking account of the definition of $\alpha_{n_{1}, n_{2}}$,

$$
\frac{\alpha_{n_{1}}}{\alpha_{N_{1}}} \rho_{N_{1}}^{n_{1}-N_{1}} \frac{\alpha_{n_{2}}}{\alpha_{N_{2}}} \rho_{N_{2}}^{n_{2}-N_{2}}
$$

and each factor is less than 1 unless $n_{1}=N_{1}$ or $n_{2}=N_{2}$, in which case it is 1 [4, pp. 319, 322]. It follows from this and (2.4) that the central index of $f\left(r_{1}, r_{2}\right)$ is $\left(N_{1}, N_{2}\right)$ and thus $(2.4)$ can be rewritten:

$$
\left|a_{n_{1}, n_{2}}\right| r_{1}^{n_{1}} r_{2}^{n_{2}} \leq \mu\left(r_{1}, r_{2}\right) \frac{\alpha_{n_{1}}}{\alpha_{N_{1}}} \rho_{N_{1}}^{n_{1}-N_{1}} \frac{\alpha_{n_{2}}}{\alpha_{N_{2}}} \rho_{N_{2}}^{n_{2}-N_{2}}, \quad n_{1} \geq 0, n_{2} \geq 0
$$

These are the main inequalities. In conformity with one-variable usage, let us call a value $\left(r_{1}, r_{2}\right)$ for which $(2.5)$ holds normal, and any other value exceptional. Evidently the normal values contain the set

$$
\begin{aligned}
&\left\{\left(t_{1} \rho_{N_{1}\left(t_{1}, t_{2}\right)}, t_{2} \rho_{N_{2}\left(t_{1}, t_{2}\right)}\right):\right.\left(N_{1}\left(t_{1}, t_{2}\right), N_{2}\left(t_{1}, t_{2}\right)\right) \\
&\text { is the central index of } \left.F\left(z_{1}, z_{2}\right)\right\} .
\end{aligned}
$$

\section{THE NORMAL SET}

The preceding derivation of the main inequalities is a straightforward duplication of the approach in the one-variable case but the estimate of the exceptional set requires something else. It happens that in two variables the nature of the normal set is such that the easier course is to deal directly with it rather than the exceptional set; in one variable both sets are unions of disjoint intervals, so there is no qualitative difference, and the estimate of the exceptional set presents itself at once analytically. Here the approach is essentially geometrical.

In considering the normal set, it is useful to represent the central index of $F\left(t_{1}, t_{2}\right)$ graphically, in the way described by Fuchs [3, p. 50]. (The one-variable analogue is given in Valiron's book [10, p. 28].) One takes the convex envelope of the points $\left(n_{1}, n_{2},-\log \left|A_{n_{1}, n_{2}}\right|\right)$, and considers the point of contact of this surface with a support plane that has, as its normal vector, $\left(\log t_{1}, \log t_{2},-1\right)$. In most cases this point is a vertex of the surface, and its coordinate pair-by which we shall mean its first two coordinates- $\left(N_{1}, N_{2}\right)$ is, as Fuchs shows, the central index of $F\left(t_{1}, t_{2}\right)$; in the remaining cases, when the support plane is parallel to one of the faces of the surface or contains one of its edges, the point is not uniquely determined, but then all corners of the associated face or edge correspond to terms of maximum modulus and the coordinate pair of one of them is the central index of $F\left(t_{1}, t_{2}\right)$.

Normal values arising from the vertex $\mathbf{V}=\left(N_{1}, N_{2},-\log \left|A_{N_{1}, N_{2}}\right|\right)$ are points $\left(t_{1} \rho_{N_{1}}, t_{2} \rho_{N_{2}}\right)$, where $\left(\log t_{1}, \log t_{2},-1\right)$ is the normal vector to any plane that supports the surface at $\mathbf{V}$. The logarithmic measure of such normal values is the plane measure of their logarithmic images-that is, of the points $\left(\log t_{1}+\log \rho_{N_{1}}, \log t_{2}+\log \rho_{N_{2}}\right)$ obtained by taking the logarithm of each component-which is the same as the measure of the set $S_{\mathbf{V}}=\left\{\left(\log t_{1}, \log t_{2}\right)\right\}$. $S_{\mathbf{V}}$ is represented by the open, convex, polygonal patch illuminated by the beam of vectors $\left(\log t_{1}, \log t_{2},-1\right)$ emanating from the origin and shining onto the plane one unit below the plane of the first two coordinates. It is a consequence of the convexity of the surface that these patches are disjoint, that they interlock and, taken together over all vertices $\mathbf{V}$, pave the plane, covering all of it apart from the boundary edges of each patch. 
The logarithmic image of the normal points is the union of the translated sets

$$
T_{\mathbf{V}}=S_{\mathbf{V}}+\left(\log \rho_{N_{1}}, \log \rho_{N_{2}}\right),
$$

taken over all vertices $\mathbf{V}$. The effect of these translations is to break up the interlocked sets $S_{\mathbf{V}}$ into nonoverlapping floes $T_{\mathbf{V}}$; the exceptional set corresponds to the vacant space introduced between them. That the sets $T_{\mathbf{V}}$ are disjoint can be seen as follows. Just as a convex surface was formed from the coefficients of the series for $F$, a similar surface can be formed from $f$, and the central index of $f\left(r_{1}, r_{2}\right)$ is the coordinate pair of the vertex at which the surface is supported by a plane with normal $\left(\log r_{1}, \log r_{2},-1\right)$. The vertex is unique if there is only one term of maximum modulus in the series, a condition that is fulfilled at any normal value $\left(r_{1}, r_{2}\right)$. Thus $T_{\mathbf{V}}$ is a subset of the beam of normals $\left(\log r_{1}, \log r_{2},-1\right)$ associated with the vertex on the surface for $f$ which has the same coordinate pair $\left(N_{1}, N_{2}\right)$ as $\mathbf{V}$, and since these beams are disjoint, so too are the sets $T_{\mathbf{V}}$.

Let us note also that if a certain part of $S_{\mathbf{V}}$ is in the first quadrant, then, since $\rho_{n} \geq 1$ for all $n$, its translate remains in the first quadrant.

From these considerations we conclude that, for any $R \geq 1$, the logarithmic measure of the normal points contained in $\Delta_{R}$, the set of (1.4), is at least $\log$ meas $\left(\Delta_{R_{0}}\right)$, where $R_{0}$ is the largest number such that, for any point $\left(t_{1}, t_{2}\right)$ in $\Delta_{R_{0}}$, the corresponding normal point $\left(t_{1} \rho_{N_{1}}, t_{2} \rho_{N_{2}}\right)$ satisfies $t_{1} \rho_{N_{1}} \leq R$ and $t_{2} \rho_{N_{2}} \leq R$.

\section{Proof of Theorem 1}

Following Hayman [4, p. 323] we choose $\alpha(t)=\int_{0}^{t} \alpha^{\prime}(t) d t$, with

$$
\begin{aligned}
\alpha^{\prime}(t) & =\frac{\varepsilon}{t \log t \log _{2} t \cdots\left(\log _{k+1} t\right)^{1+\varepsilon}}, \quad t \geq t_{0}, \\
\alpha^{\prime}(t) & =\alpha^{\prime}\left(t_{0}\right), \quad 0 \leq t \leq t_{0},
\end{aligned}
$$

where $t_{0}=e_{k+1}(1)$. Evidently

$$
\alpha(t)=C-\left(\log _{k+1} t\right)^{-\varepsilon}
$$

for all large $t$, where

$$
C=1+\frac{\varepsilon}{e_{k}(1) e_{k-1}(1) \cdots e_{1}(1)}<1+\varepsilon \text { if } k>0, \quad C=1+\varepsilon \text { if } k=0
$$

From (2.5) we have, for all normal $\left(r_{1}, r_{2}\right)$,

$$
\begin{aligned}
M\left(r_{1}, r_{2}\right) & \leq \sum_{n_{1}=0}^{\infty} \sum_{n_{2}=0}^{\infty}\left|a_{n_{1}, n_{2}}\right| r_{1}^{n_{1}} r_{2}^{n_{2}} \\
& \leq \mu\left(r_{1}, r_{2}\right) \sum_{n_{1}=0}^{\infty} \frac{\alpha_{n_{1}}}{\alpha_{N_{1}}} \rho_{N_{1}}^{n_{1}-N_{1}} \sum_{n_{2}=0}^{\infty} \frac{\alpha_{n_{2}}}{\alpha_{N_{2}}} \rho_{N_{2}}^{n_{2}-N_{2}} .
\end{aligned}
$$

Hayman has shown [4, Lemma 5 and p. 331] that

$$
\sum_{n=0}^{\infty} \frac{\alpha_{n}}{\alpha_{N}} \rho_{N}^{n-N} \sim \sqrt{2 \pi / \alpha^{\prime}(N)} \text { as } N \rightarrow \infty,
$$


so that, for some constant $K=K(k, \varepsilon)$,

$$
\sum_{n=0}^{\infty} \frac{\alpha_{n}}{\alpha_{N}} \rho_{N}^{n-N} \leq K\left\{1+N \log _{1}^{+} N \log _{2}^{+} N \cdots\left(\log _{k+1}^{+} N\right)^{1+\varepsilon}\right\}^{1 / 2}
$$

and thus

$$
\begin{aligned}
M\left(r_{1}, r_{2}\right) \leq K^{2} \mu\left(r_{1}, r_{2}\right)\{[1+ & \left.N_{1} \log _{1}^{+} N_{1} \ldots\left(\log _{k+1}^{+} N_{1}\right)^{1+\varepsilon}\right] \\
\cdot & {\left.\left[1+N_{2} \log _{1}^{+} N_{2} \cdots\left(\log _{k+1}^{+} N_{2}\right)^{1+\varepsilon}\right]\right\}^{1 / 2}, }
\end{aligned}
$$

which gives (1.6). Moreover it follows from (4.4) that (1.6) holds with $K$ replaced by any number greater than $2 \pi / \varepsilon$, provided that $N_{1}$ and $N_{2}$ are sufficiently large, which establishes part of the assertion following the statement of Theorem 1.

To proceed we need inequalities for $N_{1}$ and $N_{2}$ in terms of $\mu\left(r_{1}, r_{2}\right)$. With the harmless assumption $f(0,0)=1$, the case $n_{1}=0, n_{2}=0$ of $(2.5)$ yields

$$
\alpha_{N_{1}} \rho_{N_{1}}^{N_{1}} \alpha_{N_{2}} \rho_{N_{2}}^{N_{2}} \leq \mu\left(r_{1}, r_{2}\right)
$$

for all normal values $\left(r_{1}, r_{2}\right)$. The left-hand side of (4.6) is, from (2.1) and (2.3),

$$
\exp \left(\int_{0}^{N_{1}} t \alpha^{\prime}(t) d t+\int_{0}^{N_{2}} t \alpha^{\prime}(t) d t\right)
$$

and (it follows from our choice of $\alpha$ )

$$
\int_{0}^{x} t \alpha^{\prime}(t) d t=\frac{(1+o(1)) \varepsilon x}{\log _{1}^{+} x \log _{2}^{+} x \cdots\left(\log _{k+1}^{+} x\right)^{1+\varepsilon}},
$$

and therefore, concentrating for the moment on $N_{1}$, we obtain from (4.6)

$$
\frac{N_{1}}{\log _{1}^{+} N_{1} \log _{2}^{+} N_{1} \cdots\left(\log _{k+1}^{+} N_{1}\right)^{1+\varepsilon}} \leq \frac{K}{\varepsilon} \log \mu\left(r_{1}, r_{2}\right),
$$

for another constant $K=K(k, \varepsilon)$. If $N_{1}$ is sufficiently large, (4.7) will hold with $K$ replaced by any number greater than 1 . Taking logarithms repeatedly, we obtain

$$
\log _{1}^{+} N_{1} \leq(1+o(1)) \log _{2}^{+} \mu\left(r_{1}, r_{2}\right), \quad \log _{2}^{+} N_{1} \leq(1+o(1)) \log _{3}^{+} \mu\left(r_{1}, r_{2}\right),
$$

and so on, which, in conjunction with (4.7), gives

$$
\begin{aligned}
& N_{1} \log _{1}^{+} N_{1} \cdots\left(\log _{k+1}^{+} N_{1}\right)^{1+\varepsilon} \\
& \quad \leq \frac{K}{\varepsilon} \log _{1}^{+} \mu\left(r_{1}, r_{2}\right)\left\{\log _{2}^{+} \mu\left(r_{1}, r_{2}\right) \cdots\left(\log _{k+2}^{+} \mu\left(r_{1}, r_{2}\right)\right)^{1+\varepsilon}\right\}^{2},
\end{aligned}
$$

for a further constant $K=K(k, \varepsilon)$. This and its equivalent for $N_{2}$, when combined with (4.5), give (1.8). Since $K$ in (4.8) can be replaced by any number greater than 1 , provided that $N_{1}$ is sufficiently large, we conclude that (1.8) holds with the constant replaced by any number greater than $2 \pi / \varepsilon^{2}$, provided that both $N_{1}$ and $N_{2}$ are sufficiently large, which is the remaining part of the assertion following Theorem 1.

To estimate the exceptional set let us note that, given $R \geq 1$ and $\left(t_{1}, t_{2}\right)$ in $\Delta_{R_{0}}$, where $R_{0}=e^{-C} R$ and $C$ is given by (4.2), the corresponding normal point $\left(r_{1}, r_{2}\right)=\left(t_{1} \rho_{N_{1}}, t_{2} \rho_{N_{2}}\right)$ lies in $\Delta_{R}$, since $\rho_{j}=e^{\alpha(j)} \leq e^{C}$ for every 
$j$. It follows from the concluding remark of the preceding section that, for any $R \geq 1$, the logarithmic measure of the normal points in $\Delta_{R}$ is at least $\log$ meas $\left(\Delta_{R_{0}}\right)=\left(\log \left(e^{-C} R\right)\right)^{2}$. Equivalently, the logarithmic measure of the exceptional points in $\Delta_{R}$ is at most $\log$ meas $\left(\Delta_{R}\right)-\left(\log \left(e^{-C} R\right)\right)^{2}=2 C \log R+$ $O(1)<2(1+\varepsilon) \log R+O(1)$, using (4.2).

\section{Proof of Theorem 2}

In this case we choose

$$
\begin{aligned}
& \alpha(t)=(\lambda+\varepsilon)^{-1} t, \quad 0 \leq t \leq 1, \\
& \alpha(t)=(\lambda+\varepsilon)^{-1} \log (e t), \quad t \geq 1 .
\end{aligned}
$$

As before we have (4.3) for all normal $\left(r_{1}, r_{2}\right)$. In this case Hayman's estimate [4, Lemma 5 and p. 332] for the series on the right-hand side of (4.3) gives

$$
M\left(r_{1}, r_{2}\right) \leq 2 \pi(\lambda+\varepsilon+o(1)) \mu\left(r_{1}, r_{2}\right)\left(K+N_{1}\right)^{1 / 2}\left(K+N_{2}\right)^{1 / 2},
$$

where $K=K(\lambda, \varepsilon)$ is a constant, which is (1.9). Once again we have (4.6), which in this case yields

$$
\exp \left\{(\lambda+\varepsilon)^{-1}\left(N_{1}+N_{2}+O(1)\right)\right\} \leq \mu\left(r_{1}, r_{2}\right),
$$

so that

$$
N_{1}+N_{2} \leq(\lambda+\varepsilon+o(1)) \log \mu\left(r_{1}, r_{2}\right),
$$

and therefore

$$
\left(K+N_{1}\right)+\left(K+N_{2}\right) \leq(\lambda+\varepsilon+o(1)) \log \mu\left(r_{1}, r_{2}\right) .
$$

From the arithmetic-geometric mean inequality we deduce that

$$
\left(K+N_{1}\right)^{1 / 2}\left(K+N_{2}\right)^{1 / 2} \leq \frac{1}{2}(\lambda+\varepsilon+o(1)) \log \mu\left(r_{1}, r_{2}\right),
$$

and (1.10) follows from this combined with (5.1).

It remains to estimate the exceptional set. For any normal point $\left(r_{1}, r_{2}\right)$ we have, from (5.2),

$$
\begin{aligned}
\log \rho_{N_{1}} & =\alpha\left(N_{1}\right)=(\lambda+\varepsilon)^{-1} \log \left(e N_{1}\right) \\
& \leq(\lambda+\varepsilon+o(1))^{-1} \log _{2} \mu\left(r_{1}, r_{2}\right) \quad \text { as }\left(r_{1}, r_{2}\right) \rightarrow \infty,
\end{aligned}
$$

if $N_{1} \geq 1$, and the inequality holds also when $N_{1}=0$. Similarly

$$
\log \rho_{N_{2}} \leq(\lambda+\varepsilon+o(1))^{-1} \log _{2} \mu\left(r_{1}, r_{2}\right) .
$$

Thus, if $\left(t_{1}, t_{2}\right)$ is the point from which $\left(r_{1}, r_{2}\right)$ is derived, in the sense that $r_{1}=\rho_{N_{1}} t_{1}$ and $r_{2}=\rho_{N_{2}} t_{2}$, then

$$
\max \left\{r_{1}, r_{2}\right\} \leq \max \left\{t_{1}, t_{2}\right\}\left(\log \mu\left(r_{1}, r_{2}\right)\right)^{1 /(\lambda+\varepsilon+o(1))} \quad \text { as }\left(r_{1}, r_{2}\right) \rightarrow \infty .
$$

Now $\log \mu\left(r_{1}, r_{2}\right) \leq \log M\left(r_{1}, r_{2}\right) \leq \max \left\{r_{1}, r_{2}\right\}^{\lambda+o(1)}$, since $f$ has order $\lambda$, so

$$
\max \left\{r_{1}, r_{2}\right\} \leq \max \left\{t_{1}, t_{2}\right\}^{(\lambda+\varepsilon+o(1)) /(\varepsilon+o(1))} \text { as }\left(r_{1}, r_{2}\right) \rightarrow \infty .
$$

Hence, given $\eta>0$,

$$
\max \left\{r_{1}, r_{2}\right\} \leq \max \left\{t_{1}, t_{2}\right\}^{(\lambda+\varepsilon+\eta) / \varepsilon},
$$

for all large, normal $\left(r_{1}, r_{2}\right)$, and so for all large $\left(t_{1}, t_{2}\right)$. We conclude that, given $R \geq 1$, if $\left(t_{1}, t_{2}\right)$ is large in $\Delta_{R_{0}}$, where $R_{0}=R^{\varepsilon /(\lambda+\varepsilon+\eta)}$, then 
$\max \left\{r_{1}, r_{2}\right\} \leq R$. From the remark at the end of $\S 3$ we deduce that the logarithmic measure of the normal set in $\Delta_{R}$ is at least $\left\{\frac{\varepsilon}{\lambda+\varepsilon+\eta} \log R\right\}^{2}$. Thus the exceptional set has upper logarithmic density no more than $1-\left(\frac{\varepsilon}{\lambda+\varepsilon+\eta}\right)^{2}$, and the conclusion of Theorem 2 follows since $\eta>0$ is arbitrary.

\section{Two EXAMPLES}

The intention of the examples is to establish the assertions made in the introduction to do with the constants of Theorems 1 and 2. Both examples are of the form $f\left(z_{1}, z_{2}\right)=F\left(z_{1}\right) F\left(z_{2}\right)$, where $F$ is a certain entire function of one variable; in both cases we shall rely on Hayman's analysis of $F$.

Hayman has shown $[4$, p. 334] that, given an integer $j \geq 0$, there is an entire function $F(z)$ for which

$$
M(r, F)=(1+o(1)) \mu(r, F)\left\{2 \pi N \log _{1}^{+} N \cdots \log _{j+1}^{+} N\right\}^{1 / 2},
$$

as $r \rightarrow \infty$, where $M(r, F)$ is the maximum modulus, $\mu(r, F)$ is the maximum term and $N=N(r, F)$ is the central index. Now, with $f\left(z_{1}, z_{2}\right)=F\left(z_{1}\right) F\left(z_{2}\right), M\left(r_{1}, r_{2}, f\right)=M\left(r_{1}, F\right) M\left(r_{2}, F\right), \mu\left(r_{1}, r_{2}, f\right)=$ $\mu\left(r_{1}, F\right) \mu\left(r_{2}, F\right)$, and $\mathbf{N}\left(r_{1}, r_{2}, f\right)=\left(N\left(r_{1}, F\right), N\left(r_{2}, F\right)\right)$, so

$$
\begin{aligned}
M\left(r_{1}, r_{2}, f\right)= & (2 \pi+o(1)) \mu\left(r_{1}, r_{2}, f\right)\left\{N_{1} \log _{1}^{+} N_{1} \cdots \log _{j+1}^{+} N_{1}\right\}^{1 / 2} \\
& \cdot\left\{N_{2} \log _{1}^{+} N_{2} \cdots \log _{j+1}^{+} N_{2}\right\}^{1 / 2},
\end{aligned}
$$

as $\left(r_{1}, r_{2}\right) \rightarrow \infty$. Thus, taking $j>k$, where $k$ is the number of Theorem 1 , we deduce that $\varepsilon$ cannot be replaced by 0 in (1.6).

Again, with the same notation, Hayman has shown [4, p. 332] that, given $\lambda>0$, there exists an entire function $F$ such that

$$
M(r, F)=(1+o(1)) \mu(r, F)\{2 \pi \lambda N\}^{1 / 2}
$$

and also

$$
N(r, F)=(\lambda+o(1)) \log \mu(r, F)=(c+o(1)) r^{\lambda}
$$

as $r \rightarrow \infty$, where $c$ is a constant. Thus, with $f\left(z_{1}, z_{2}\right)=F\left(z_{1}\right) F\left(z_{2}\right)$,

$$
M\left(r_{1}, r_{2}, f\right)=(2 \pi \lambda+o(1)) \mu\left(r_{1}, r_{2}, f\right)\left\{N_{1} N_{2}\right\}^{1 / 2}
$$

as $\left(r_{1}, r_{2}\right) \rightarrow \infty$, and we conclude that $\varepsilon$ cannot be negative in (1.9). Finally, in view of $(6.1)$, we shall have, if $0<\varepsilon<\lambda$,

$$
M\left(r_{1}, r_{2}, f\right)>\pi(\lambda-\varepsilon)^{2} \mu\left(r_{1}, r_{2}, f\right) \log \mu\left(r_{1}, r_{2}, f\right),
$$

for all large $\left(r_{1}, r_{2}\right)$ in a small angle about the line $r_{1}=r_{2}$; and the logarithmic measure of the intersection of such an angle with $\Delta_{R}$ is asymptotic to a constant times $\log R$.

\section{REFERENCES}

1. P. C. Fenton, Some results of Wiman-Valiron type for integral functions of finite lower order, Ann. of Math. (2) 103 (1976), 237-252.

2. A A note on the Wiman-Valiron method, Proc. Edinburgh Math. Soc. 37 (1993), 53-55.

3. W. H. J. Fuchs, A look at Wiman-Valiron theory, Complex Analysis, Kentucky 1976, Lecture Notes in Math., vol. 599, Springer, 1977. 
4. W. K. Hayman, The local growth of power series: a survey of the Wiman-Valiron method, Canad. Math. Bull. 17 (1974), 317-358.

5. T. Kövari, On theorems of Pólya and Turan, J. Analyse Math. 6 (1958), 323-332.

6. (1961), 71-109.

7. P. C. Rosenbloom, Probability and entire functions, Studies in Mathematical Analysis (G. Szegö et al., eds.), Stanford Univ., 1963.

8. A. Schumitzky, Wiman-Valiron theory for entire functions of several complex variables, Ph.D. Thesis, Cornell Univ., 1965.

9. __ A probabilistic approach to the Wiman-Valiron theory for entire functions of several complex variables, Complex Variables 13 (1989), 85-98.

10. G. Valiron, Lectures on the general theory of integral functions, Chelsea, 1949.

Department of Mathematics, University of Otago, Dunedin, New Zealand 